\title{
Clinical and Translational Imaging two years after take-off
}

\author{
Giovanni Lucignani
}

Published online: 6 February 2015

(c) Italian Association of Nuclear Medicine and Molecular Imaging 2015

\begin{abstract}
"Consider well the seed that gave you birth: you were not made to live your lives as brutes, but to be followers of worth and knowledge".
\end{abstract}

\section{Dante Alighieri, Inferno, XXVI, 119-120}

This issue of Clinical and Translational Imaging (CTI) marks the beginning of the third year of its publication, and the stakeholders of the journal deserve a short report on the activities carried out and on the developments and perspectives of the journal.

CTI was launched in January 2013 and the interest in the journal has steadily increased over the first 2 years thanks to a publication strategy aiming at identifying relevant topics in molecular imaging worthy of review.

An international editorial board including a panel of renowned experts in different areas of clinical imaging and basic science, mostly with an $h$ index over 20, has characterized the journal from the beginning. The editorial board members support the journal in the peer-review process and many of them have been actively involved as guest editors of published and forthcoming issues.

In 2014, 16 new members from different countries joined the board, further intensifying the international blend. European representatives now account for $73 \%$ of

G. Lucignani $(\bowtie)$

Department of Biomedical Sciences (DISS) and Center of Molecular and Cellular Imaging (IMAGO), University of Milan, Milan, Italy

e-mail: giovanni.lucignani@unimi.it

G. Lucignani

Unit of Nuclear Medicine, Department of Diagnostic Services, San Paolo Hospital, Via Antonio di Rudinì 8, 20142 Milan, Italy board members, while $22 \%$ are based in America and $4 \%$ in Asia.

Although the journal publishes invited contributions, all submitted papers undergo a strict peer review process that requires a broad panel of reviewers. To this end, more than 200 colleagues were invited to participate in the peerreview process in 2014, $50 \%$ of whom completed their task of reviewing the 54 submissions received. Following the revision process $9 \%$ of manuscripts were accepted without changes, $63 \%$ after minor modifications, and $23 \%$ were subjected to major revision by the authors at the request of the referees. A few manuscripts were rejected because they did not fall within the scope of CTI (5\%).

Some new features have been introduced in Editorial Manager in order to improve the peer review process: new reviewers' grids have been implemented to assist the peerreview process, while detailed instructions for reviewers have been published on springer.com. Classification categories have been introduced for reviewers and authors to correctly identify appropriate reviewers for submitted manuscripts.

The six issues published in 2014 were guest edited by 12 scientists from Europe, America and Asia and included 49 articles (an increase of $15 \%$ over 2013), $69 \%$ from Europe, $27 \%$ from America, $2 \%$ from Asia and $2 \%$ from Australia.

Issue contents published in 2013 and 2014 were made freely accessible online, in order to maximize immediate article visibility and citation, through Springer's online platform SpringerLink (http://link.springer.com).

The number of downloads increased by $70 \%$ from more than 9,000 full-text articles in 2013 to more than 15,000 in 2014, competing with consolidated journals in the field. Over the same time span the number of citations has also steadily increased. To date the journal has received 158 
citations in Google Scholar. Thomson Reuters-ISI recorded 10 articles in 2013 and 37 articles in 2014 that cited CTI, yielding 14 external cites in 2013 and 58 in 2014, not including self-cites.

The analysis of the main bibliometric indexes at the end of 2014 indicates an $h$ index of 7 (compared with 4 in 2013).

The visibility of the journal has also been increased following several successful applications to abstracting services including EMBASE, Google Scholar, ProQuest, Academic Search, ChemWeb, INIS Atomindex, OCLC, Summon by ProQuest. An application to Scopus was submitted in October 2014 and the journal is still under evaluation. Since the overall content, quality and importance of published papers seem to be in line with requirements for inclusion in Medline's databases, an application will be submitted in the spring of 2015 .

In 2014 special attention was devoted to ethical issues. As a member of the Committee on Publication Ethics (COPE), CTI follows the COPE guidelines dealing with potential acts of misconduct and malicious misrepresentations of research data. To ensure that accepted principles of ethical and professional conduct have been followed, all published papers include information regarding sources of funding, potential conflicts of interest (financial or nonfinancial), and declarations of adherence to the ethical principles required when conducting research. Moreover, iThenticate software was made available to the journal to screen for plagiarism.

A limit on the number of co-authors' names is now recommended in order to prevent non-substantial authorship; authors whose names appear on the submission are requested to confirm that they have contributed sufficiently to the scientific work and therefore share collective responsibility and accountability for the results.

Along with the editorial and the review papers each issue of CTI now includes a Spotlight contribution, a short text in the form of an editorial intended to spark discussions on emerging and less widely discussed matters related to the topic of the issue.

Since the journal receives submissions from non-English-speaking countries, intensive language editing has been performed on accepted articles over the past 2 years. However, due to an appreciable improvement in article quality, also following the broadening of authorship to native speakers of English, for the sake of a quicker turnover time from submission to publication it was decided to move the journal to a standard copyediting process as for most international journals. Authors who are not entirely confident in English can take advantage of the Edanz Springer Author Academy, an editing service for authors of scientific manuscripts.

Several 2015 issue topics have already been scheduled and guest editors, along with most of the authors, have been invited. He topics of the issues in 2015 will be amyloid imaging, sentinel lymph nodes, musculoskeletal pathology, PET in lymphoma, imaging of myocardial innervation, and dose reduction in nuclear medicine. Planning for the year 2016 is also under way to ensure timely publication of the six issues per year.

Over the past 2 years CTI was published both online and in print. However, following the international trend towards online journal usage, starting with the first issue of 2015 the journal will be published online only.

The year 2015 marks also the end of the free availability of the journal online, in accordance with the contract between AIMN and Springer. Two types of access will be available from now on: to individual subscribers, including members of the AIMN through the AIMN website, and to the entire scientific community through consortia. In 2013 and 2014 the journal was included in 400 consortium deals covering $90 \%$ of libraries worldwide, including emerging countries (China, India, Brazil, Turkey). This wide exposure, as well as visibility and usage, of CTI will be maintained and expanded in the future.

The supportive structure and organization, the efficient work of the editorial office, has been fundamental for the work of the authors, the reviewers and the board; the commitment of the community that has grown up around the journal and of the editorial staff is the key to future success.

Based on the evidence of sincere commitment on the part of the editorial board, reviewers and authors, and on the evidence demonstrating the increasing impact of the journal in the scientific arena, the prospects for CTI appear promising although more work will be needed to improve quality and guarantee the journal's continuity and growth.

\section{G. Lucignani}

Editor-in-Chief 